OPEN ACCESS

Edited by:

Gian Luigi Gigli,

University of Udine, Italy

Reviewed by:

Anastasia Bougea,

National and Kapodistrian University

of Athens, Greece

Samir Abu-Rumeileh,

Ulm University Medical

Center, Germany

*Correspondence:

Shuqin Zhan

shqzhan@hotmail.com

Specialty section:

This article was submitted to

Sleep Disorders,

a section of the journal

Frontiers in Neurology

Received: 30 November 2021

Accepted: 14 January 2022

Published: 18 February 2022

Citation:

Yuan Y, Zhang Y, Cheng Y, Hou Y,

Huang Z, Ma J, Li N and Zhan S

(2022) Cerebrospinal Fluid TNF- $\alpha$ and

Orexin in Patients With Parkinson's

Disease and Rapid Eye Movement

Sleep Behavior Disorder

Front. Neurol. 13:826013.

doi: 10.3389/fneur.2022.826013

\section{Cerebrospinal Fluid TNF- $\alpha$ and Orexin in Patients With Parkinson's Disease and Rapid Eye Movement Sleep Behavior Disorder}

\author{
Yuan Yuan ${ }^{1,2,3}$, Yimeng Zhang ${ }^{1,2,3}$, Yueyang Cheng ${ }^{1,2,3}$, Yue Hou ${ }^{1,2,3}$, Zhaoyang Huang ${ }^{1,2,3}$, \\ Jinghong $\mathrm{Ma}^{1}$, Ning $\mathrm{Li}^{1,2,3}$ and Shuqin Zhan ${ }^{1,2,3 *}$ \\ ${ }^{1}$ Department of Neurology, Xuanwu Hospital, Capital Medical University, Beijing, China, ${ }^{2}$ Beijing Key Laboratory of \\ Neuromodulation, Beijing, China, ${ }^{3}$ Beijing Institute of Brain Disorders, Collaborative Innovation Center for Brain Disorders, \\ Capital Medical University, Beijing, China
}

Background: Parkinson's disease (PD) pathological changes begin before motor symptoms appear. Rapid eye movement sleep behavior disorder (RBD) has the highest specificity and predictive value of any marker of prodromal PD. Tumor necrosis factor $\alpha(T N F-\alpha)$ plays a part in the pathology of PD and disease conversion in isolated RBD (iRBD). TNF can also directly impair the hypocretin system in mice in vivo. As a result, we intend to investigate the effect of TNF- $\alpha$ on orexin levels in PD patients with RBD.

Method: Participants were recruited from the Department of Neurology of Xuanwu Hospital, Capital Medical University to engage in assessments on motor symptoms, sleep, cognition, etc. Then we collected blood and cerebrospinal fluid of all patients and 10 controls' cerebrospinal fluid. The levels of TNF- $\alpha$ in the serum and cerebrospinal fluid, as well as the level of orexin in the cerebrospinal fluid, were measured in the patients.

Results: The difference in TNF- levels in cerebrospinal fluid and serum between the three groups were not statistically significant. The levels of orexin in the three groups were not significantly lower than in the control group. UPDRS-III scores were significantly higher in the PD+RBD and PD-RBD groups than in the RBD group. There was no statistically significant difference in $\mathrm{H}-\mathrm{Y}$ stages, $\mathrm{PSQ}$, or ESS scores between the PD+RBD and PD-RBD groups.

Conclusion: Our findings suggest that TNF- $\alpha$ may not have a significant effect on the orexinergic system in patients with Parkinson's disease and RBD. As a result, it is necessary to investigate the changes in TNF- $\alpha$ and orexin levels in different disease stages and to enlarge the sample size to determine whether TNF- $\alpha$ affects the function of the orexin system, which may be related to the occurrence of RBD and disease progression in Parkinson's disease.

Keywords: rapid eye movement sleep behavior disorder, Parkinson's disease, cerebrospinal fluid, TNF- $\alpha$, orexin 


\section{INTRODUCTION}

Parkinson's disease (PD) is a common neurodegenerative disease characterized clinically by resting tremor, bradykinesia, rigidity, and postural balance disorder. The presence of fibrillar aggregates, known as Lewy bodies (LBs), in which $\alpha$-synuclein is a major constituent, is a histopathological hallmark of Parkinson's disease (1). With the in-depth study of the disease, nonmotor symptoms (NMS) have received increasing attention and have become a new research hotspot in Parkinson's disease. Hyposmia, autonomic dysfunction, anxiety and depression, cognitive impairment, and sleep disturbances are a few examples.

Rapid eye movement (REM) sleep behavior disorder (RBD) is a parasomnia characterized by loss of muscle atonia with abnormal dream-enacting behavior during REM sleep (2), which can result in injuries to these individuals and their bed partners. Idiopathic or isolated RBD (iRBD) is defined as $\mathrm{RBD}$ in the absence of any relevant neurological disorder or other precipitating factors (2). According to a longitudinal study, the majority of patients are eventually diagnosed with synucleinopathies such as Parkinson's disease (PD), dementia with Lewy bodies (DLB), and multiple system atrophy (MSA) (3). Multicenter prospective cohort studies have shown that over $60 \%$ of iRBD developed overt $\alpha$-synucleinopathies in a decade or more and the phenoconversion rate was $6.25 \%$ per year (4). A meta-analysis confirmed a risk of more than $90 \%$ at 14 years (5). Furthermore, RBD confirmed by polysomnography (PSG) has by far the highest specificity and predictive value of any prodromal PD marker (6). According to Braak, $\alpha$-synuclein deposition begins in the anterior olfactory nucleus, the dorsal motor nucleus of the vagus (7), implying that the pathological changes of PD begin before motor symptoms manifest. As a result, the clinical diagnosis for Parkinson's disease treatment may be delayed. For this reason, identifying reliable biomarkers is critical.

Orexins, also known as hypocretins, are neuropeptides produced by the hypothalamus and play a part in metabolism, feeding, reward, addiction, and sleep-wake control (8). According to the LU-SAPER model (9), orexin may indirectly participate in the innervation of spinal motor neurons by the sublaterodorsal tegmental nucleus (SLD) via fiber projections to the lateral pontine tegmentum (LPT), influencing REM atonia during sleep.

TNF- $\alpha$, a potent pro-inflammatory cytokine, is recognized as an important mediator of neuroinflammation in the brain (10). TNF- $\alpha$ produced in large by activated microglia contributes to neuroinflammatory processes in a variety of neurological disorders (11). TNF- $\alpha$ has been shown to play a role in the pathology of Parkinson's disease (12) and disease conversion in iRBD (13). According to a study, TNF can impair the hypocretin system directly in vivo in mice. The research also suggests that repeated TNF challenge induces RBD-like behavior and sleep dysfunction in mice, as well as a decrease in learning, cognition, and memory (14).

As a result, we wonder if there are any links between the levels of TNF- $\alpha$ and orexin in cerebrospinal fluid (CSF) in patients with Parkinson's disease and rapid eye movement sleep behavior disorder. We recruited patients to investigate the above-mentioned correlations. TNF- $\alpha$ and orexin levels in cerebrospinal fluid and serum TNF- $\alpha$ were measured and compared.

\section{METHOD}

\section{Participants}

Patients with Parkinson's disease and iRBD were recruited for this study from the Department of Neurology of Xuanwu Hospital, Capital Medical University. To assess subjects on motor symptoms, sleep, cognition and emotion, we used Unified Parkinson's Disease Rating Scales part III (UPDRS-III), Hoehn-Yahr (H-Y) stage, Pittsburgh sleep quality index (PSQI), Epworth sleepiness scale (ESS), REM Sleep Behavior Disorder Questionnaire-Hong Kong (RBDQ-HK), Cognitive impairment uses Montreal Cognitive Assessment (MoCA), Hamilton rating scale for anxiety (HAMA) and Hamilton rating scale for depression (HAMD). Patients were classified in iRBD, PD with $\mathrm{RBD}(\mathrm{PD}+\mathrm{RBD}), \mathrm{PD}$ without RBD (PD-RBD) groups. The following were the inclusion criteria for Parkinson's disease patients: (1) diagnosis refer to MDS clinical diagnostic criteria for Parkinson's disease (15), (2) age range of 40 to 80 years. The diagnosis of RBD refers to the International Classification of Sleep Disorders, Third Version (ICSD-3). Exclusion criteria were as follows: (1) those with parkinsonism from a cause other than Parkinson's disease, (2) those with a history of head injury, brain tumor, encephalitis, stroke; and abnormal electroencephalography (EEG) suggesting epilepsy, (3) those who take sleeping pills or antipsychotics, use sedative-hypnotic drugs or alcohol to help sleep and are unable to withdraw, (4) those who are unable to complete overnight PSG. The following criteria were used to select 10 age-matched control subjects: no neurodegenerative diseases, no sleep disorders, and no epilepsy. The overnight video-polysomnograph examination was performed and analyzed for 145 patients.

\section{Clinical Assessment}

Patients were subjected to a thorough neurological examination, which included the Unified Parkinson's Disease Rating Scale part III (UPDRS III) and the Hoehn-Yahr (H-Y) stage for motor symptoms. The REM Sleep Behavior Disorder QuestionnaireHong Kong (RBDQ-HK) was used to assess RBD. Daytime sleepiness and sleep quality were assessed using the Epworth sleepiness scale (ESS) and the Pittsburgh sleep quality index (PSQI). Cognitive impairment was evaluated using the Montreal Cognitive Assessment (MoCA). To quantify anxiety and depressive symptom severity, the Hamilton rating scale for anxiety (HAMA) and Hamilton rating scale for depression (HAMD) were used.

\section{Video-Polysomnograph}

The Compumedics E-series polysomnography monitoring system, manufactured in Australia by Compumedics, was used to monitor patients' sleep throughout the night. The monitoring content includes EEG (electrodes installed in accordance with the international 10-20 system, respectively F3, F4, C3, C4, O1-A2, O2-A1), electrooculogram, chin, and both lower limbs EMG, mouth Nasal airflow (pressure sensing and thermal 
sensing), chest and abdomen breathing, electrocardiogram, and blood oxygen saturation.

\section{CSF and Serum Samples Collection and Analysis}

CSF was collected from 20 patients via lumbar puncture standardized procedures and placed in siliconized polypropylene tubes. The samples were then centrifuged for $10 \mathrm{~min}$ at $4^{\circ} \mathrm{C}$ within $30 \mathrm{~min}$ of blood collection. After centrifugation, the extracted samples were stored at $-80^{\circ} \mathrm{C}$. The level of orexin was measured by radioimmunoassay. TNF- $\alpha$ level was measured by chemiluminescent immunoassay. TNF- $\alpha$ was evaluated using the IMMULITE/IMMULITE 1000 TNF- $\alpha$ kits (Siemens Healthcare Diagnostics, Llanberis, UK) according to manufacturer instructions. The average intra- and inter-assay coefficients of variation were $3.5 \%$ and $6.5 \%$, respectively.

\section{Statistical Analysis}

We used mean, standard deviation, median, range, and quartile to present data. The SPSS version 25 software package was used to perform all statistical analyses. One-way ANOVA was used to analyze data with a normal distribution. We used the
Kruskal Wallis $\mathrm{H}$ test for non-normal distribution data. The chisquare test was used to analyze data for categorical variables. Pearson correlation analysis was used to assess the statistical relationship between TNF- $\alpha$ and orexin levels. A $p$-value of $<0.05$ was considered statistically significant.

\section{RESULTS}

\section{Demographics and Clinical Assessments}

According to the inclusion criteria listed above, a total of 145 patients were studied, including $38 \mathrm{PD}$ patients with $\mathrm{RBD}, 55$ $\mathrm{PD}$ patients without RBD, and $52 \mathrm{iRBD}$ patients. Clinical data were compared between the $\mathrm{PD}+\mathrm{RBD}, \mathrm{PD}-\mathrm{RBD}$, and $\mathrm{iRBD}$ groups (motor symptoms, non-motor symptoms: cognition, emotion, and sleep). There was no significant difference in age or gender ratio among the included patients. UPDRS-III scores were significantly higher in the $\mathrm{PD}+\mathrm{RBD}$ and $\mathrm{PD}-\mathrm{RBD}$ groups than in the iRBD group $[22(14.89,34.50)$ vs. $17(15.45,26.50)$ vs. $1(0,2.25), P<0.001]$. There was no remarkable difference in $\mathrm{H}-\mathrm{Y}$ stages, PSQI, ESS scores between PD+RBD and PDRBD groups. However, RBDQ-HK scores in the iRBD group were significantly higher than those in the other groups with

TABLE 1 | Demographics and clinical tests including Unified Parkinson's Disease Rating Scale part III (UPDRS-III) and Hoehn-Yahr (H-Y) stage scores, Pittsburgh sleep quality index (PSQI), Epworth sleepiness scale (ESS), REM Sleep Behavior Disorder Questionnaire-Hong Kong (RBDQ-HK), Montreal Cognitive Assessment (MoCA), Hamilton rating scale for anxiety (HAMA), Hamilton rating scale for depression (HAMD) in Parkinson's disease (PD), isolated rapid eye movement sleep behavior disorder ( $\mathrm{RBD}$ ) and PD with RBD subjects.

\begin{tabular}{|c|c|c|c|c|c|c|c|}
\hline & \multicolumn{2}{|r|}{ PD+RBD } & \multicolumn{2}{|r|}{ PD-RBD } & \multicolumn{2}{|r|}{ iRBD } & \multirow[t]{2}{*}{$P$-value } \\
\hline & $N$ & & $N$ & & $N$ & & \\
\hline Age (mean $\pm S D$ ), years & 38 & $64.35 \pm 9.83$ & 55 & $59.94 \pm 9.17$ & 52 & $63.33 \pm 8.88$ & 0.053 \\
\hline Gender (Male number/\%) & 38 & $22.00 / 57.89 \%$ & 55 & $29.00 / 52.73 \%$ & 52 & $33.00 / 63.46 \%$ & 0.532 \\
\hline UPDRS-III [M, (P25, P75)] & 9 & $22.00(14.89,34.50)$ & 5 & $17.00(15.45,26.50)$ & 10 & $1.00(0,2.25)$ & $<0.001^{\mathrm{bc}}$ \\
\hline $\mathrm{H}$-Y stage (mean, range) & 36 & $2.06,1-4$ & 48 & $2.03,1-3$ & - & - & - \\
\hline PSQI [M, (P25, P75)] & 9 & $5.00(4.50,7.86)$ & 5 & $8.00(5.86,16.50)$ & 10 & $6.00(2.75,6.72)$ & 0.115 \\
\hline ESS (mean \pm SD) & 37 & $4.50 \pm 3.55$ & 43 & $5.00 \pm 4.24$ & 48 & $4.88 \pm 4.29$ & 0.854 \\
\hline RBDQ-HK (mean \pm SD) & 37 & $22.38 \pm 18.74$ & 43 & $15.66 \pm 14.28$ & 48 & $34.07 \pm 19.16$ & $<0.001^{\mathrm{ac}}$ \\
\hline MoCA (mean \pm SD) & 21 & $21.01 \pm 4.28$ & 21 & $21.77 \pm 4.38$ & 20 & $23.87 \pm 3.11$ & 0.067 \\
\hline HAMA (mean \pm SD) & 12 & $18.17 \pm 5.82$ & 6 & $14.92 \pm 6.76$ & 19 & $12.24 \pm 6.00$ & $0.040^{\mathrm{b}}$ \\
\hline HAMD (mean \pm SD) & 12 & $13.92 \pm 3.98$ & 6 & $14.08 \pm 4.59$ & 19 & $8.92 \pm 8.87$ & 0.106 \\
\hline TIB [M, (P25, P75)], min & & $500.50(518.00,578.50)$ & & $545.75(521.35,567.60)$ & & $546.50(523.35,578.35)$ & 0.773 \\
\hline TST [M, (P25, P75)], min & & $365.00(313.00,457.00)$ & & $380.75(305.00,427.50)$ & & $385.50(329.10,443.25)$ & 0.483 \\
\hline SE [M, (P25, P75)], \% & & $69.90(55.60,81.70)$ & & $68.55(56.15,78.95)$ & & $71.80(60.60,82.55)$ & 0.477 \\
\hline SL [M, (P25, P75)], min & & $13.50(6.50,32.50)$ & & $23.75(12.00,50.85)$ & & $23.25(7.60,42.00)$ & 0.133 \\
\hline WASO [M, (P25, P75)], min & & $102.00(57.00,154.00)$ & & $101.00(77.50,154.60)$ & & $95.00(50.85,154.00)$ & 0.559 \\
\hline N1 [M, (P25, P75)], \%TST & 38 & $9.00(5.80,14.80)$ & 55 & $8.75(5.10,15.10)$ & 52 & $9.50(6.40,13.80)$ & 0.919 \\
\hline N2 [M, (P25, P75)], \%TST & & $49.20(37.10,56.60)$ & & $50.60(38.90,60.60)$ & & $52.20(45.00,58.75)$ & 0.612 \\
\hline N3 [M, (P25, P75)], \%TST & & $21.90(10.50,34.60)$ & & $21.95(15.30,31.75)$ & & $18.20(12.65,27.20)$ & 0.305 \\
\hline R [M, (P25, P75)], \%TST & & $14.70(12.70,22.60)$ & & $13.40(9.05,19.00)$ & & $17.80(14.25,21.40)$ & $0.011^{c}$ \\
\hline AHI [M, (P25, P75)], /hr & & $6.20(2.30,17.50)$ & & $2.50(0.25,8.50)$ & & $6.15(1.75,19.45)$ & 0.054 \\
\hline PLMSI [M, (P25,P75)], /hr & & $0(0,49.30)$ & & $0(0,46.00)$ & & $0(0,59.10)$ & 0.859 \\
\hline
\end{tabular}

N, number; SD, standard deviation; $M$, median; $P 25,25 \%$ percentile values; $P 75,75 \%$ percentile values.

${ }^{a} p$-values significant differences were found between $P D+R B D$ and $P D-R B D$ groups.

${ }^{b} p$-values significant differences were found between $P D+R B D$ and iRBD groups.

${ }^{c} p$-values Significant differences were found between $P D-R B D$ and iRBD groups. 
statistical significance $(22.38 \pm 18.74$ vs. $15.66 \pm 14.28$ vs. $34.07 \pm 19.16, P<0.001)$. Although there was no statistically significant difference, the MoCA score in the $\mathrm{PD}+\mathrm{RBD}$ group was lower than the PD-RBD group (21.01 \pm 4.28 vs. 21.77 \pm 4.38 ). The PD+RBD group's HAMA score was significantly higher than the iRBD group's $(18.17 \pm 5.82$ vs. $14.92 \pm 6.76$, $P=0.004)$. Nonetheless, there was no discernible difference in HAMD scores between the three groups. Table 1 shows the demographics and clinical scores of study participants, as well as their comparison.

\section{Biomarker Data}

In this study, no significant difference was observed in the serum TNF- $\alpha$ levels among the three groups $(5.73 \pm 0.73$ vs. $6.01 \pm 1.31$ vs. $4.51 \pm 0.45 \mathrm{pg} / \mathrm{ml}, P=0.773$ ). Also, no significant difference was observed in the CSF TNF- $\alpha$ levels between the three groups $(4.20 \pm 0.37$ vs. $4.47 \pm 0.58$ vs. $6.28 \pm 2.20 \mathrm{pg} / \mathrm{ml}, P=0.368)$. CSF orexin levels were abnormal $(<200 \mathrm{pg} / \mathrm{ml})$ in all PD+RBD, PD$\mathrm{RBD}$ and iRBD patients $(177.69 \pm 46.04,177.31 \pm 29.40,166.23$ $\pm 40.62 \mathrm{pg} / \mathrm{ml})$. Yet the orexin levels of the three groups were not significantly lower than the control group. Furthermore, there

TABLE 2 | Biomarkers including tumor necrosis factor-alpha (TNF- $\alpha$ ) in serum and cerebrospinal fluid (CSF), orexin in cerebrospinal fluid in the three groups.

\begin{tabular}{|c|c|c|c|c|c|}
\hline & PD+RBD $(n=9)$ & PD-RBD $(n=4)$ & iRBD $(n=7)$ & Control $(n=10)$ & $P$-value \\
\hline Serum TNF- $\alpha$ (mean \pm SD), pg/mL & $5.73 \pm 0.73$ & $6.01 \pm 1.31$ & $4.51 \pm 0.45$ & NA & 0.773 \\
\hline CSF TNF- $\alpha$ (mean \pm SD), pg/mL & $4.20 \pm 0.37$ & $4.47 \pm 0.58$ & $6.28 \pm 2.20$ & NA & 0.368 \\
\hline CSF orexin (mean $\pm \mathrm{SD}$ ), pg/mL & $177.69 \pm 46.04$ & $177.31 \pm 29.40$ & $166.23 \pm 40.62$ & $218.83 \pm 43.36$ & 0.224 \\
\hline
\end{tabular}

A

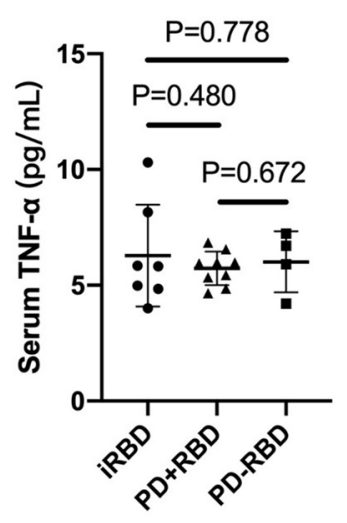

C

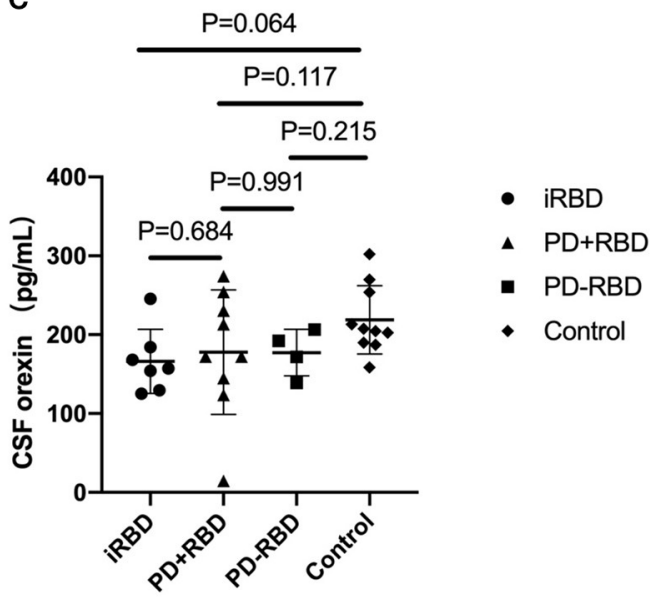

B

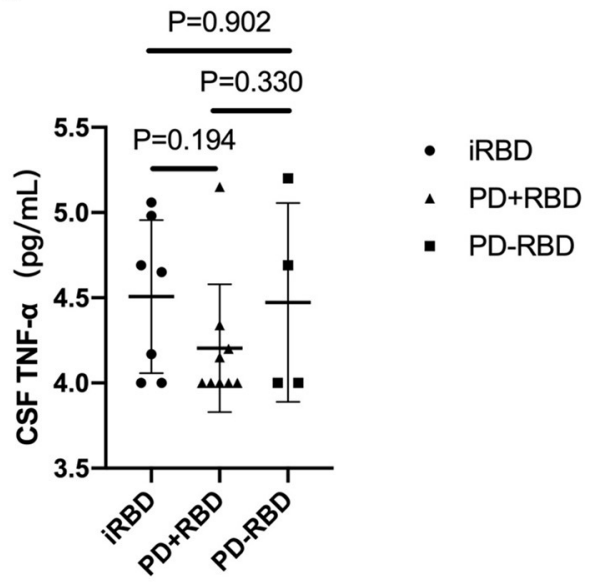

FIGURE 1 | The levels of TNF- $\alpha$ in serum and cerebrospinal fluid (CSF) in PD patients with RBD, PD patients without RBD, and isolated RBD patients (A,B). The levels of orexin in CSF in the four groups (C). Bars represent the mean values, and T bars indicate the standard deviations. 
was no significant correlation between the levels of TNF- $\alpha$ and orexin $(P=0.647)$ (see Table 2, Figure 1).

\section{DISCUSSION}

This study explored biomarkers in serum and CSF in iRBD and Parkinson's disease patients. Also, we attempt to untangle the relationship between inflammatory marker TNF- $\alpha$ and the orexinergic system. We speculated that TNF- $\alpha$ may not induce the onset and progression of neurodegenerative diseases by acting on the orexin system.

Evidence suggests that CSF and blood biomarkers closely reflecting the pathophysiology of Parkinson's disease, such as major intermediary filament of astrocytes glial fibrillary acidic protein (GFAP) (16), a marker of astroglial activation YKL-40 (17) and proinflammatory cytokines tumor necrosis factor-alpha, IL-6, and IL-12 (12), may have diagnostic and prognostic value.

Inflammation, specifically glial activation, has been linked to the progressive degenerative process in Parkinson's disease. Increased level of TNF- $\alpha$ in CSF from PD patients has been observed in previous studies $(18,19)$. Furthermore, as with the prodromal phenotype of Parkinson's disease, the neurodegenerative process of iRBD is mediated by TNF- $\alpha$ similarly (13), suggesting that a-synuclein pathologies have already existed in $\mathrm{iRBD}$ and play a role in the activation of microglia. However, it is still unclear how it affects the onset and progression of the disease.

Interestingly, TNF- $\alpha$ was found to downregulate orexin levels and exhibit increased REM sleep electromyographic activity in a previously published animal study via a protein degradation mechanism (14). As we all know, orexin deficiency is a common feature of narcolepsy type one patients (20). It is unknown whether orexin has any effect on neurodegenerative disease. According to Luppi et al., orexin is indirectly involved in spinal motor neuron innervation in the lower part of the dorsolateral tegmental nucleus (SLD) via fiber projections to the pontine tegmental nucleus, and orexin deficiency can cause decreased neuronal excitability in SLD, resulting in muscle atonia in REM sleep (9). This study sheds light on clinical research. However, the results of current studies on the level of orexins in CSF from Parkinson's disease and iRBD patients vary. Several studies have found that the number of orexin neurons in the postmortem hypothalamus and the level of orexin in ventricular CSF are significantly lower than in controls (21-23). Despite the fact that the differences were not statistically significant, our study found that orexin levels in the three groups decreased when compared to controls. Orexin plays a neuroprotective role in Parkinson's disease via a variety of mechanisms, including maintaining the firing of nigral dopamine neurons (24). We believe that the lower levels of orexin found in our study indicate that the orexinergic system dysfunction plays a role in the pathogenesis of Parkinson's disease. Furthermore, functional abnormalities may have occurred during the prodromal stage of Parkinson's disease. However, orexin levels may have no relation to disease progression or phenotype. Another study suggests that high levels of orexin in Parkinson's disease are linked to the loss of
REM muscle atonia (25). According to the study, orexin enhances muscle activity via both direct effects on spinal motor neurons and indirect effects on locus coeruleus neurons. Furthermore, some studies show no significant reduction in orexin levels in CSF from Parkinson's disease and iRBD patients $(26,27)$. The difference in CSF orexin levels between their results and our study could be explained by the CSF collection method. Another possible explanation for these disparities is that the patients in the studies were sampled differently. TNF- $\alpha$ levels in serum and CSF of PD patients with RBD were not significantly different from those of other groups in this study, implying that TNF- $\alpha$ may not have a significant effect on the orexinergic system.

Although there was no statistically significant difference, the UPDRS-III score in the PD+RBD group was higher than the PD-RBD group, which is consistent with previous research that PD patients with RBD have more severe motor impairment (28). Except for the fact that the RBDQ-HK score of the PD+RBD and iRBD group was higher than that of the PD-RBD group, we found no significant difference among the three groups in sleep scale scores. Despite the lack of a significant difference, the mean value of MoCA in the PD+RBD group was lower than PDRBD group. Many studies have found that PD patients with RBD have a higher risk of cognitive impairment (29). The link between RBD and cognitive dysfunction could be due to the brainstem nuclei involved in RBD mediating cognition (30). The study found no more depression in the PD+RBD group than in the other two groups, which is consistent with previous research (31). Furthermore, our study discovered that PD patients with RBD were more anxious than iRBD patients, which may result from comorbidity. Furthermore, a study indicates that dysfunctions in the raphe nucleus and the locus ceruleus cause anxiety in Parkinson's disease patients (32). It has also been reported that PD patients with RBD are more likely to experience anxiety and depression, and it is believed that the presence of RBD causes anxiety and depression in PD patients, which can further affect the sleep quality of patients (33). Sleep architecture did not differ significantly among the three groups, except for a higher proportion of REM sleep in iRBD patients compared to the PD-RBD group.

There are several limitations to this study. First, in patients who do not complain of abnormal behaviors in their sleep, the object of inquiry about the PSQI and RBDQ-HK should be the family members or caregivers of the patient rather than the patient himself. The resulting scale scores more accurately reflect the patients' actual RBD severity. Second, this study didn't further divided patients with or without therapeutic medication into subgroups. After the symptomatic improvement, the results of scales such as UPDRS, RBDQ-HK, HAMA may be different from those obtained prior to medication, and fail to reflect the actual situation, causing bias in the results. Third, PD patients with RBD were not further classified as to whether RBD appeared before or after motor symptoms of PD. We wonder if the time node at which RBD first appears may be related to the severity of neuroinflammatory reactions in the central and peripheral nervous system. Finally, the sample size of this study is small, and more patients should be recruited in the study in the future. 
In conclusion, our study investigated biomarkers of phenoconversion in Parkinson's disease and iRBD, as well as the relationship between inflammatory factor TNF- $\alpha$ and orexinergic system in clinical aspect, which might be useful for risk stratification of disease conversion. In the future, the effects of TNF- $\alpha$ and orexin on Parkinson's disease should be studied and discussed in a larger sample size.

\section{DATA AVAILABILITY STATEMENT}

The raw data supporting the conclusions of this article will be made available by the authors, without undue reservation.

\section{ETHICS STATEMENT}

The studies involving human participants were reviewed and approved by Xuanwu Hospital, Capital Medical University

\section{REFERENCES}

1. Wakabayashi K, Tanji K, Odagiri S, Miki Y, Mori F, Takahashi H.The Lewy body in Parkinson's disease and related neurodegenerative disorders. Mol Neurobiol. (2013) 495-508. doi: 10.1007/s12035-012-8 280-y

2. Hogl B, Stefani A, Videnovic A. Idiopathic REM sleep behaviour disorder and neurodegeneration-an update. Nat Rev Neurol. (2018) 40-55. doi: 10.1038/nrneurol.2017.157

3. Iranzo A, Fernández-Arcos A, Tolosa $\mathrm{E}$, Serradell $\mathrm{M}$, Molinuevo JL, Valldeoriola F, et al. Neurodegenerative disorder risk in idiopathic REM sleep behavior disorder: study in 174 patients. PLoS ONE. (2014) 9:e89741. doi: 10.1371/journal.pone.0089741

4. Postuma RB, Iranzo A, Hu M, Högl B, Boeve BF, Manni R, et al. Risk and predictors of dementia and parkinsonism in idiopathic REM sleep behaviour disorder: a multicentre study. Brain. (2019) 142:744-59. doi: 10.1093/brain/awz030

5. Galbiati A, Verga L, Giora E, Zucconi M, Ferini-Strambi L. The risk of neurodegeneration in REM sleep behavior disorder: a systematic review and meta-analysis of longitudinal studies. Sleep Med Rev. (2019) 43:37-46. doi: 10.1016/j.smrv.2018.09.008

6. Postuma RB, Berg D. Advances in markers of prodromal Parkinson disease. Nat Rev Neurol. (2016) 12:622-34. doi: 10.1038/nrneurol.2016.152

7. Braak H, Del Tredici K, Rüb U, de Vos RA, Jansen Steur EN, Braak E. Staging of brain pathology related to sporadic Parkinson's disease. Neurobiol Aging. (2003) 24:197-211. doi: 10.1016/S0197-4580(02)00065-9

8. Ohno K, Sakurai T. Orexin neuronal circuitry: role in the regulation of sleep and wakefulness. Front Neuroendocrinol. (2008) 29:70-87. doi: 10.1016/j.yfrne.2007.08.001

9. Lu J, Sherman D, Devor M, Saper CB. A putative flip-flop switch for control of REM sleep. Nature. (2006) 441:589-94. doi: 10.1038/nature04767

10. Frankola KA, Greig NH, Luo W, Tweedie D. Targeting TNF- $\alpha$ to elucidate and ameliorate neuroinflammation in neurodegenerative diseases. CNS Neurol Disord Drug Targets. (2011) 10:391-403. doi: 10.2174/187152711794653751

11. Olmos G, Lladó J. Tumor necrosis factor alpha: a link between neuroinflammation and excitotoxicity. Mediators Inflamm. (2014) 2014:861231. doi: 10.1155/2014/861231

12. Béraud D, Hathaway HA, Trecki J, Chasovskikh S, Johnson DA, Johnson JA, et al. Microglial activation and antioxidant responses induced by the Parkinson's disease protein $\alpha$-synuclein. J Neuroimmune Pharmacol. (2013) 8:94-117. doi: 10.1007/s11481-012-9401-0

13. Kim R, Lee JY, Kim HJ, Kim YK, Nam H, Jeon B. Serum TNFalpha and neurodegeneration in isolated REM sleep behavior disorder.
Institutional Review Board. The patients/participants provided their written informed consent to participate in this study.

\section{AUTHOR CONTRIBUTIONS}

YY, YZ, YC, YH, ZH, JM, NL, and SZ: conceived and designed the experiments. YY, YZ, YC, YH, ZH, and JM: performed the experiments. YY, YZ, YC, and YH: analyzed the data. YY, YZ, YC, NL, and SZ: wrote the paper. All authors read and approved the content.

\section{FUNDING}

This work was supported by grants from the National Natural Science Foundation of China (Grant Numbers: 81571294 and 2017YFC0909102) and the Foundation of Chinese Sleep Research Society Hansoh Project.
Parkinsonism Relat Disord. (2020) 81:1-7. doi: 10.1016/j.parkreldis.2020. 09.041

14. Zhan S, Che P, Zhao XK, Li N, Ding Y, Liu J, et al. Molecular mechanism of tumour necrosis factor alpha regulates hypocretin (orexin) expression, sleep and behaviour. J Cell Mol Med. (2019) 23:6822-34. doi: 10.1111/jcmm.14566

15. Postuma RB, Berg D, Stern M, Poewe W, Olanow CW, Oertel W, et al. MDS clinical diagnostic criteria for Parkinson's disease. Mov Disord. (2015) 30:1591-601. doi: 10.1002/mds.26424

16. Lotankar S, Prabhavalkar KS, Bhatt LK. Biomarkers for Parkinson's Disease: Recent Advancement. Neurosci Bull. (2017) 33:585-97. doi: 10.1007/s12264-017-0183-5

17. Hall S, Janelidze S, Surova Y, Widner H, Zetterberg H, Hansson O. Cerebrospinal fluid concentrations of inflammatory markers in Parkinson's disease and atypical parkinsonian disorders. Sci Rep. (2018) 8:13276. doi: 10.1038/s41598-018-31517-z

18. Iwaoka K, Otsuka C, Maeda T, Yamahara K, Kato K, Takahashi K, et al. Impaired metabolism of kynurenine and its metabolites in CSF of parkinson's disease. Neurosci Lett. (2020) 14:134576. doi: 10.1016/j.neulet.2019.134576

19. Karpenko MN, Vasilishina AA, Gromova EA, Muruzheva ZM, Miliukhina IV, Bernadotte A. Interleukin-1beta, interleukin-1 receptor antagonist, interleukin-6, interleukin-10, and tumor necrosis factor-alpha levels in CSF and serum in relation to the clinical diversity of Parkinson's disease. Cell Immunol. (2018) 327:77-82. doi: 10.1016/j.cellimm.2018.02.011

20. Bassetti CLA, Adamantidis A, Burdakov D, Han F, Gay S, Kallweit U, et al. Narcolepsy-clinical spectrum, aetiopathophysiology, diagnosis and treatment. Nat Rev Neurol. (2019) 519-39. doi: 10.1038/s41582-019-0 226-9

21. Drouot X, Moutereau S, Nguyen JP, Lefaucheur JP, Créange A, Remy $\mathrm{P}$, et al. Low levels of ventricular CSF orexin/hypocretin in advanced PD. Neurology. (2003) 61:540-3. doi: 10.1212/01.WNL.0000078194.53 210.48

22. Fronczek R, Overeem S, Lee SY, Hegeman IM, van Pelt J, van Duinen SG, et al. Hypocretin (orexin) loss in Parkinson's disease. Brain. (2007) 130:1577-85. doi: 10.1093/brain/awm090

23. Thannickal TC, Lai YY, Siegel JM. Hypocretin (orexin) cell loss in Parkinson's disease. Brain. (2007) 130:1586-95. doi: 10.1093/brain/awm097

24. Berhe DF, Gebre AK, Assefa BT. Orexins role in neurodegenerative diseases: From pathogenesis to treatment. Pharmacol Biochem Behav. (2020) 194:172929. doi: 10.1016/j.pbb.2020.172929

25. Bridoux A, Moutereau S, Covali-Noroc A, Margarit L, Palfi S, Nguyen JP, et al.Ventricular orexin-A (hypocretin-1) levels correlate with rapid-eyemovement sleep without atonia in Parkinson's disease. Nat Sci Sleep. (2013) 5:87-91. doi: 10.2147/NSS.S41245 
26. Yasui K, Inoue Y, Kanbayashi T, Nomura T, Kusumi M, Nakashima K. CSF orexin levels of Parkinson's disease, dementia with Lewy bodies, progressive supranuclear palsy and corticobasal degeneration. J Neurol Sci. (2006) 250:120-3. doi: 10.1016/j.jns.2006.08.004

27. Anderson KN, Vincent A, Smith IE, Shneerson JM. Cerebrospinal fluid hypocretin levels are normal in idiopathic REM sleep behaviour disorder. Eur J Neurol. (2010) 17:1105-7. doi: 10.1111/j.1468-1331.2010.0 2954.x

28. Lee JE, Kim KS, Shin HW, Sohn YH. Factors related to clinically probable REM sleep behavior disorder in Parkinson disease. Parkinsonism Relat Disord. (2010) 16:105-8. doi: 10.1016/j.parkreldis.2009. 08.005

29. Liu H, Ou R, Wei Q, Hou Y, Cao B, Zhao B, et al. Rapid eye movement behavior disorder in drug-naive patients with Parkinson's disease. J Clin Neurosci. (2019) 59:254-8. doi: 10.1016/j.jocn.2018.07.007

30. Vazey EM, Aston-Jones G. The emerging role of norepinephrine in cognitive dysfunctions of Parkinson's disease. Front Behav Neurosci. (2012) 6:48. doi: 10.3389/fnbeh.2012.00048

31. Vendette M, Gagnon JF, Décary A, Massicotte-Marquez J, Postuma RB, Doyon J, et al. REM sleep behavior disorder predicts cognitive impairment in Parkinson disease without dementia. Neurology. (2007) 69:1843-9. doi: 10.1212/01.wnl.0000278114.14096.74

32. Dissanayaka NN, White E, O'Sullivan JD, Marsh R, Pachana NA, Byrne GJ. The clinical spectrum of anxiety in Parkinson's disease. Mov Disord. (2014) 29:967-75. doi: 10.1002/mds. 25937

33. Mahale RR, Yadav R, Pal PK. Rapid eye movement sleep behaviour disorder in women with Parkinson's disease is an underdiagnosed entity. J Clin Neurosci. (2016) 28:43-6. doi: 10.1016/j.jocn.2015.08.046

Conflict of Interest: The authors declare that the research was conducted in the absence of any commercial or financial relationships that could be construed as a potential conflict of interest.

Publisher's Note: All claims expressed in this article are solely those of the authors and do not necessarily represent those of their affiliated organizations, or those of the publisher, the editors and the reviewers. Any product that may be evaluated in this article, or claim that may be made by its manufacturer, is not guaranteed or endorsed by the publisher.

Copyright $\odot 2022$ Yuan, Zhang, Cheng, Hou, Huang, Ma, Li and Zhan. This is an open-access article distributed under the terms of the Creative Commons Attribution License (CC BY). The use, distribution or reproduction in other forums is permitted, provided the original author(s) and the copyright owner(s) are credited and that the original publication in this journal is cited, in accordance with accepted academic practice. No use, distribution or reproduction is permitted which does not comply with these terms. 